\title{
Diffusion Tensor Imaging Features of the Auditory Pathways in Patients With Vestibular Schwannoma After Gamma Knife Radiosurgery
}

Dilek H. Cesme ${ }^{1}$, Alpay Alkan ${ }^{1}$, Mehmet Ali Gultekin ${ }^{1}$, Lutfullah Sari ${ }^{1}$, Gokberk Alkan ${ }^{2}$, Ahmet Kaya ${ }^{1}$, Alpaslan Mayadagli ${ }^{3}$, Kerime Akdur ${ }^{3}$, Omer Uysal ${ }^{4}$, Mustafa A. Hatiboglu ${ }^{5}$

1. Department of Radiology, Faculty of Medicine, Bezmialem Vakif University, Istanbul, TUR 2. Otorhinolaryngology, Abdurrahman Yurtaslan Oncology Training and Research Hospital, Ankara, TUR 3. Radiation Oncology, Faculty of Medicine, Bezmialem Vakif University, Istanbul, TUR 4. Biostatistics, Faculty of Medicine, Bezmialem Vakif University, Istanbul, TUR 5. Neurosurgery, Faculty of Medicine, Bezmialem Vakif University, Istanbul, TUR

Corresponding author: Dilek H. Cesme, dhcesme@bezmialem.edu.tr

\section{Abstract}

\section{Objective}

In this study, we aimed to investigate whether there is any change in diffusion tensor imaging (DTI) parameters in ipsilateral and contralateral auditory pathways after Gamma Knife radiosurgery (GKR) in patients with vestibular schwannoma (VS) and the relationship between radiosurgery variables.

\section{Methods}

Sixty-six patients were evaluated with MRI and DTI before and after GKR. The apparent diffusion coefficient (ADC) and fractional anisotropy (FA) were measured from the bilateral lateral lemniscus (LL), inferior colliculus (IC), medial geniculate body (MGB), and Heschl's gyrus (HG).

\section{Results}

There was no significant difference in ADC and FA values obtained from bilateral LL, IC, and MGB before and after radiosurgery. However, there was a significant difference between pretreatment and postradiosurgery contralateral HG ADC values. The ADC values obtained from the contralateral HG and IC positively correlated with the duration after radiosurgery. As the duration after radiosurgery increases, the difference between the ADC values obtained from ipsilateral and contralateral HG also increases.

\section{Conclusion}

The high $\mathrm{ADC}$ values in the contralateral $\mathrm{HG}$ after radiosurgery may indicate microstructural alterations such as demyelination and axonal loss. Radiation exposure doses to the brainstem and cochlea are the most important factors that can cause microstructural damage to the auditory pathways. When planning radiosurgery, extreme care should be taken to prevent the harmful effects of radiation on the auditory pathways.

Review began 03/21/2021 Review ended 03/24/2021 Published 03/27/2021

\section{(c) Copyright 2021}

Cesme et al. This is an open access article distributed under the terms of the Creative Commons Attribution License CC-BY 4.0., which permits unrestricted use, distribution, and reproduction in any medium, provided the original author and source are credited.
Categories: Radiation Oncology, Radiology, Neurosurgery

Keywords: vestibular schwannoma, auditory pathways, gamma knife radiosurgery, radiosurgery, diffusion tensor imaging

\section{Introduction}

Vestibular schwannomas (VS) are benign neoplasms of Schwann cell origin. The most common symptom of the condition is unilateral neurosensory hearing loss. Additionally, dizziness, tinnitus, imbalance, headache, paresthesia, and facial paralysis complaints are also seen [1,2].

Treatment options for VS include observation, microsurgery, or radiosurgery [2-4]. Currently, Gamma Knife radiosurgery (GKR) is the preferred treatment method for small and medium-sized tumors. The aim of radiosurgery treatment is to permanently restrain the growth of VS [5]. Brainstem and cochlear radiation dose parameters have critical importance in planning radiosurgery treatment. The higher the radiation dose to which the brainstem is exposed, the greater the risk of hearing loss [6-11]. Hearing loss develops due to the toxic effect of radiation in the brainstem nuclei and cochlea $[6,7,11]$. When planning stereotactic radiosurgery for VS, especially for large lesions, the cochlear nerve will always be involved as it cannot be separated from the superior and inferior vestibular.

The auditory pathways consist of the lateral lemniscus (LL), inferior colliculus (IC), medial geniculate body (MGB), and Heschl's gyrus (HG). Fibers from the brainstem cochlear nuclei extend into the ipsilateral or 
contralateral superior olivary nucleus and reach the IC via the LL. Efferent fibers extend from the IC through the MGB in the thalamus to the auditory cortex in the HG $[12,13]$.

Diffusion tensor imaging (DTI) is an advanced neuroimaging method used to investigate white matter microstructure integrity and connectivity [6]. Fractional anisotropy (FA) and apparent diffusion coefficient (ADC) and are the most frequently used parameters in DTI. FA values provide important data about myelination, fiber density, and axon diameter in white matter. The decrease in FA may occur due to the loss of regular anisotropic structures, possibly due to a disruption of the cytoarchitecture. While ADC independently measures the direction of total water diffusion in tissues, it also provides information about tissue cellularity and nucleus-cytoplasm ratio. Increased ADC values are thought to be associated with increased extracellular fluid production and myelin disorders due to a decrease in the number of cells or axons $[6,14-16]$.

DTI studies that evaluate auditory pathways such as LL, IC, MGB, and HG in patients with VS treated with radiosurgery are scarce [6]. In this study, we aimed to evaluate whether there is any change in DTI parameters in auditory pathways after radiosurgery in patients with VS and to investigate the correlation between radiosurgery variables and DTI parameters.

\section{Materials And Methods}

Our study was approved by the Bezmialem Vakıf University Clinical Research Ethics Committee (54022451050.05.04/2020). We retrospectively collected the data of the subjects who underwent GKR treatment for VS between 2013 and 2020 at our University Hospital. Sixty-six patients (40 males, 26 females; mean age: 54.65 \pm 13 years; age range: $30-77$ years) with VS who underwent MRI and DTI imaging before and after treatment were included in the study. Patients with a history of microsurgical operation in the cerebellopontine angle and those without a follow-up MRI after radiosurgery were excluded.

\section{Magnetic resonance imaging}

The subjects with unilateral VS who were considered for GKR treatment were evaluated with a 1.5T MRI system (Avanto, Siemens Healthineers, Erlangen, Germany). The applied MRI sequences included axial and coronal fluid-attenuated inversion recovery (FLAIR) [repetition time (TR): $8000 \mathrm{~ms}$, echo time (TE): $90 \mathrm{ms,}$ inversion time (TI): $2500 \mathrm{~ms}$ ], sagittal, axial T2 turbo spin-echo (TSE) (TR: $4500 \mathrm{~ms}$, TE: $90 \mathrm{~ms}$ ), and axial T1 spin-echo (SE) (TR: $550 \mathrm{~ms}$, TE: $14 \mathrm{~ms}$ ) weighted images. T1 images with contrast in the axial, sagittal, and coronal planes were obtained [IV gadolinium-diethylenetriaminepentaacetic acid (Gd-DTPA)]. 3D T1 magnetization-prepared rapid acquisition with gradient echo (MPRAGE) images with and without contrast were added to our study.

All subjects were evaluated with DTI parameters as standard protocol [single-shot SE echo-planar, TR/TE: 6000/89 ms, field of view (FOV): 230 x $230 \mathrm{~mm}$, matrix: 128 x 256, and thickness: $5 \mathrm{~mm}$ ]. Thirty different diffusion gradient directions were used $\left(b=0 \mathrm{~s} / \mathrm{mm}^{2}\right.$ and $\left.b=1000 \mathrm{~s} / \mathrm{mm}^{2}\right)$. ADC and FA maps were created by processing DTI data on a workstation (Leonardo console, software version 2.0; Siemens). Axial colorencoded FA map images were taken as a reference while placing the regions of interest (ROI) in the auditory pathways. Four pixel ROI sizes were placed manually. Placement of all ROIs was performed simultaneously by an experienced radiologist. Follow-up images were obtained 3-60 $(19.8 \pm 15.5)$ months after the baseline images taken before radiosurgery.

\section{Gamma Knife protocol}

Treatment planning was carried out using the Gamma Knife 4C model and its software version 10.1.1 (Elekta, Stockholm, Sweden). A Leksell stereotactic head frame was placed, and images of volumetric MRI sequences 3D T2 and 3D T1 MPRAGE were taken. All patients were treated with an isodose of 50\%: the median treatment dose was $12.5 \mathrm{~Gy}$ (range: 11.5-13 Gy), the median maximum brainstem dose was $10.4 \mathrm{~Gy}$ (range: $0.7-15.1 \mathrm{~Gy}$ ), the median brainstem volume receiving a radiation dose of $10 \mathrm{~Gy}$ (BS V10) was $0.1 \mathrm{cc}$ (range: 0-0.55 cc), the median maximum cochlear dose was $5.8 \mathrm{~Gy}$ (range: $1.4-12.1 \mathrm{~Gy}$ ), and the mean cochlear dose was 3.1 Gy (range: 0.9-8.3 Gy) (Figure 1). No additional neurological deficits were detected in the follow-up MRIs after radiosurgery. The ADC and FA values of LL, IC, MGB, and HG were measured before and after radiosurgery (Figures $2 a-2 d$ ). Moreover, the relationship between radiosurgery treatment variables such as the brainstem and cochlear dose and ADC and FA values was investigated. 


\section{Cureus}

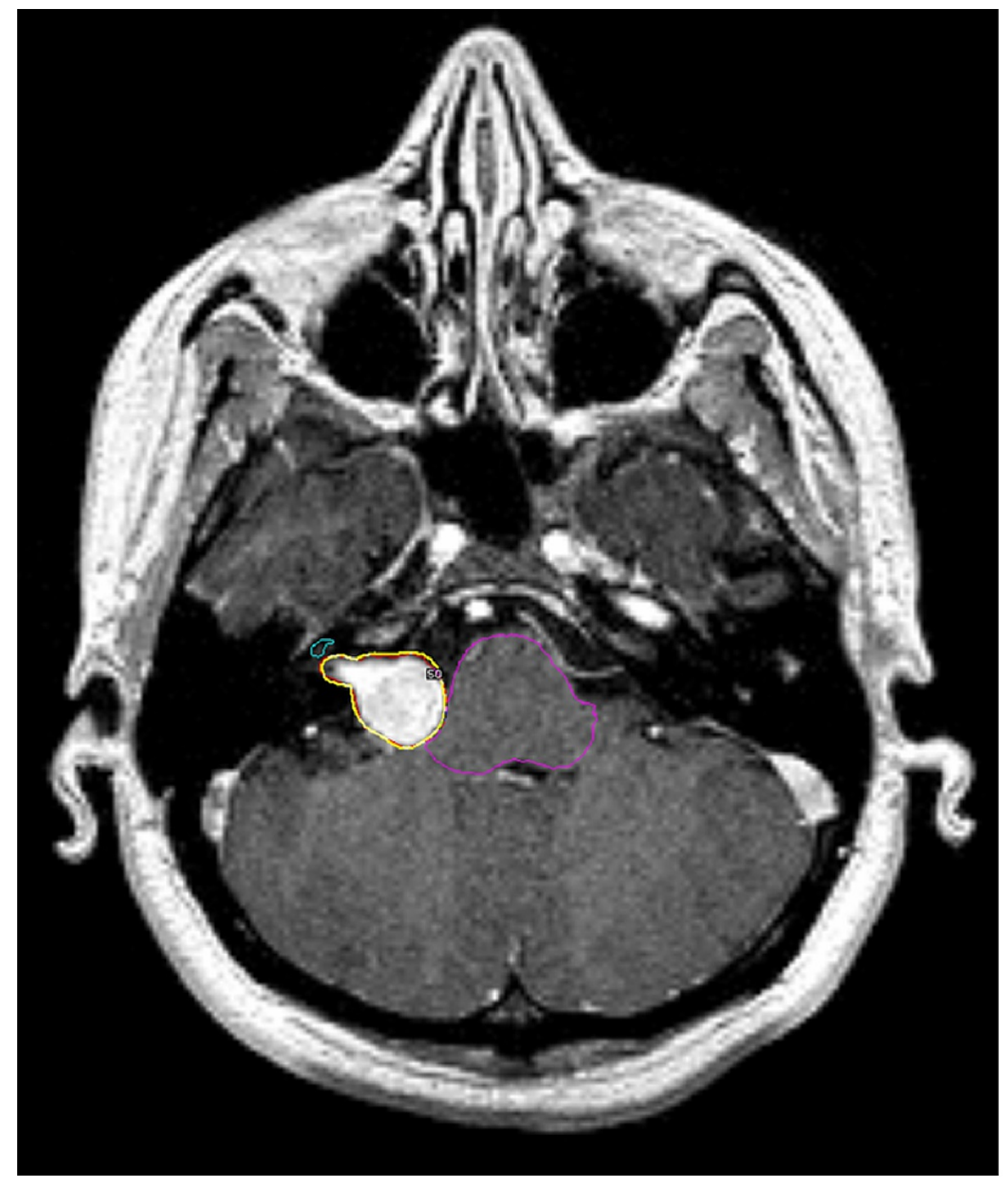

FIGURE 1: Radiosurgery planning in a case of vestibular schwannoma

The boundaries of the brainstem and cochlea (colored outlines) were defined to calculate the exposure dose 


\section{Cureus}

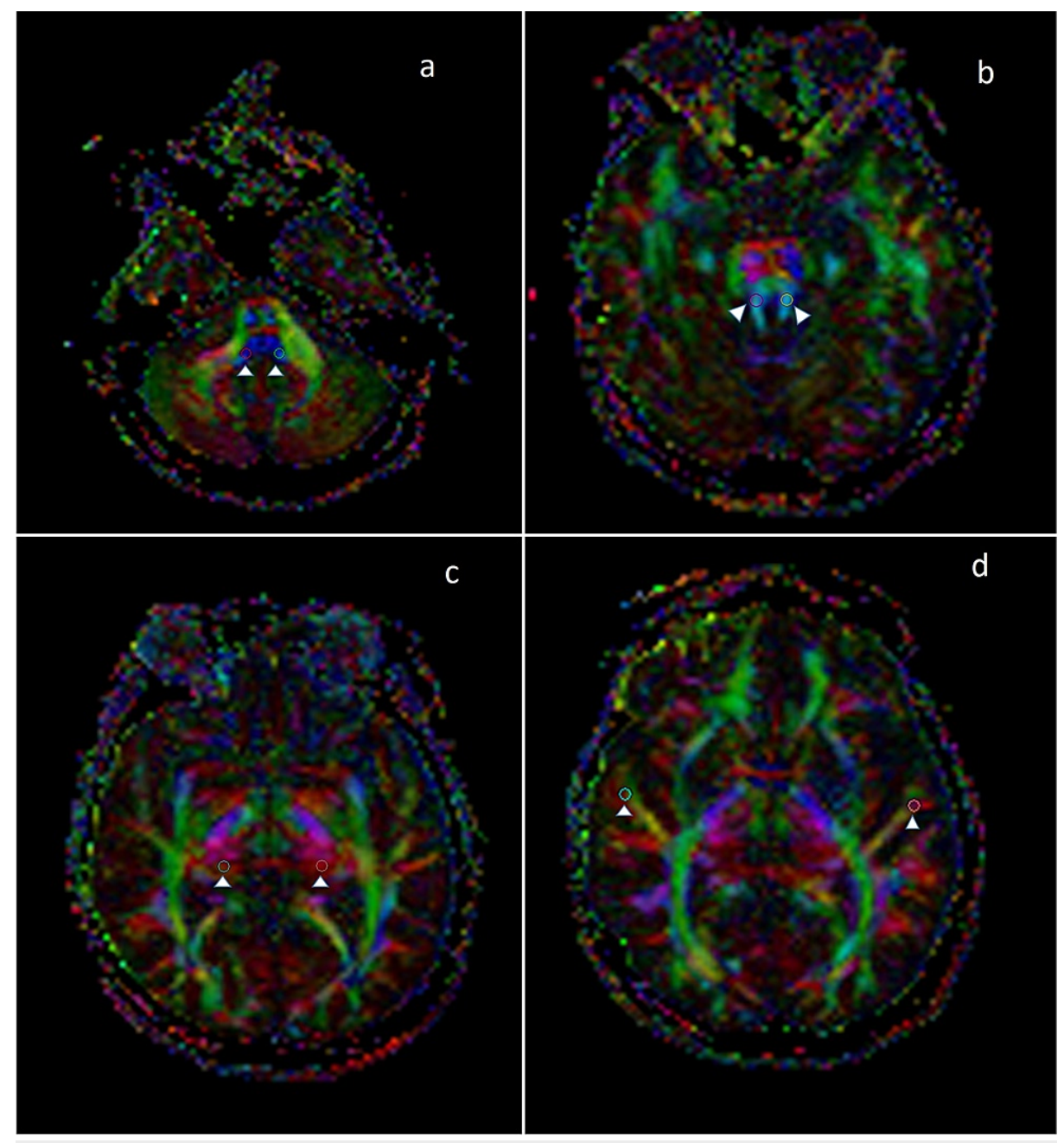

\section{FIGURE 2: Right-sided vestibular schwannoma}

The color FA maps show ROI placement at lateral lemniscus (a) (arrowheads), inferior colliculus (b) (arrowheads), medial geniculate body (c) (arrowheads), and Heschl's gyrus (d) (arrowheads)

FA: fractional anisotropy; ROI: region of interest

\section{Statistical analysis}

Statistical data were evaluated using SPSS Statistics v19.0 (IBM, Armonk, NY). The Kolmogorov-Smirnov test was used to determine whether the data were suitable for normal distribution. Parametric tests were applied since they show normal distribution. A paired t-test was used to compare the FA and ADC values obtained from the auditory pathways before and after radiosurgery. Pearson's correlation test was used to investigate the relationship between DTI parameters obtained from auditory pathways and GKR treatment variables. A $\mathrm{p}$-value of $<0.05$ was considered statistically significant.

\section{Results}

There was a significant difference in LL FA values between the ipsilateral and contralateral sides in the pretreatment period $(\mathrm{p}=0.001)$. Contralateral LL FA values were lower than ipsilateral ones in the pretreatment period. However, there was no significant difference in ADC and FA values obtained from bilateral LL, IC, and MGB before and after radiosurgery.

There was a significant difference between pretreatment and post-radiosurgery contralateral HG ADC values $(\mathrm{p}=0.002$ ). The ADC values obtained from the contralateral HG and IC positively correlated with the duration after radiosurgery $(\mathrm{r}=0.265, \mathrm{p}=0.03$ and $\mathrm{r}=0.241, \mathrm{p}=0.05$, respectively). As the duration after radiosurgery increased, the difference between the ADC values obtained from ipsilateral and contralateral $\mathrm{HG}$ also increased $(\mathrm{r}=0.282, \mathrm{p}=0.02)$.

As the maximum brainstem radiation dose increased, the difference between the contralateral HG FA values 
before and after radiosurgery also increased $(\mathrm{r}=0.247, \mathrm{p}=0.04)$. Ipsilateral MGB ADC values were positively correlated with the maximum cochlear dose $(\mathrm{r}=0.300, \mathrm{p}=0.01)$ and the mean cochlear dose $(\mathrm{r}=0.269, \mathrm{p}=0.02)$.

DTI parameters obtained from the auditory pathways before and after radiosurgery are presented in Table 1 .

\begin{tabular}{|c|c|c|c|c|c|c|c|}
\hline \multirow{2}{*}{\multicolumn{2}{|c|}{ Locations }} & \multicolumn{3}{|l|}{ ADC } & \multicolumn{3}{|l|}{ FA } \\
\hline & & Pre-GKR & Post-GKR & P-value & Pre-GKR & Post-GKR & P-value \\
\hline \multirow{2}{*}{ LL } & Ipsilateral & $0.778 \pm 0.75$ & $0.777 \pm 0.61$ & 0.98 & $0.620 \pm 0.10$ & $0.616 \pm 0.10$ & 0.79 \\
\hline & Contralateral & $0.771 \pm 0.63$ & $0.789 \pm 0.75$ & 0.10 & $0.581 \pm 0.11$ & $0.598 \pm 0.13$ & 0.21 \\
\hline \multirow{2}{*}{ IC } & Ipsilateral & $0.783 \pm 0.58$ & $0.777 \pm 0.63$ & 0.75 & $0.680 \pm 0.10$ & $0.708 \pm 0.12$ & 0.06 \\
\hline & Contralateral & $0.780 \pm 0.60$ & $0.777 \pm 0.74$ & 0.72 & $0.674 \pm 0.11$ & $0.707 \pm 0.13$ & 0.06 \\
\hline \multirow{2}{*}{ MGB } & Ipsilateral & $0.783 \pm 0.59$ & $0.797 \pm 0.85$ & 0.35 & $0.448 \pm 0.85$ & $0.436 \pm 0.85$ & 0.39 \\
\hline & Contralateral & $0.785 \pm 0.62$ & $0.795 \pm 0.75$ & 0.38 & $0.441 \pm 0.88$ & $0.442 \pm 0.95$ & 0.93 \\
\hline \multirow{2}{*}{$H G$} & Ipsilateral & $0.795 \pm 0.92$ & $0.809 \pm 0.68$ & 0.36 & $0.299 \pm 0.75$ & $0.282 \pm 0.96$ & 0.27 \\
\hline & Contralateral & $0.772 \pm 0.69$ & $0.806 \pm 0.70$ & 0.002 & $0.316 \pm 0.90$ & $0.307 \pm 0.83$ & 0.58 \\
\hline
\end{tabular}

TABLE 1: The ADC and FA values measured before and after radiosurgery from ipsilateral and contralateral sides in patients with VS

LL: lateral lemniscus; IC: inferior colliculus; MGB: medial geniculate body; HG: Heschl's gyrus; GKR: Gamma Knife radiosurgery; ADC: apparent diffusion coefficient $\left(10^{-3} \mathrm{~mm}^{2} / \mathrm{sec}\right)$; FA: fractional anisotropy; VS: vestibular schwannoma

\section{Discussion}

VS accounts for approximately 6-8\% of all brain tumors. The main goal of the treatment of VS should be functional preservation of the facial, trigeminal, and cochlear nerves rather than complete removal of the tumor. Radiosurgery is the primarily preferred treatment method as it is associated with over $95 \%$ remission, low complication rate, preservation of neurological functions, and good quality of life in patients [5,17-20].

Hearing stimuli originate from the cochlear branch of the eighth cranial nerve. The ventral and dorsal cochlear nuclei located in the brainstem are very important anatomical functional structures for auditory pathways. The postsynaptic fibers are called LL. All of the fibers initiating from the cochlear nuclei are not crossed but a few of them ascend as ipsilateral lemniscus lateralis. These fibers then intermingle into the IC, the center of hearing reflexes in the mesencephalon. The fibers starting from the IC then reach the auditory cortex (HG) after synapse at MGB. The contralateral pathways have a much more important function in the processing of auditory stimuli. Also, 20-30\% of the fibers that reach the auditory cortex come from the ipsilateral side and $70-80 \%$ come from the contralateral side $[12,13]$.

DTI is a promising imaging technique used to characterize microstructural changes or differences in neuropathological events [6,12,14-16]. It can enable the identification of auditory neural tracts. Decreased FA values may suggest white matter damage and axonal degeneration. It is considered that increased ADC values may be associated with increased extracellular fluid production and myelin disorders due to a decrease in the number of cells or axons [21]. ADC values may be affected in tissue damage such as edema, degeneration, and necrosis [21]. Radiation-induced changes in the auditory pathways can be detected in patients with VS treated with radiosurgery $[6,8,9,22]$. In our study, contralateral LL FA values were lower than ipsilateral in the pretreatment period. The low FA values in the contralateral LL can be attributed to the microstructural alterations in the white matter tract depending on the anatomical course of the auditory pathway in the pre-radiosurgery period.

DTI studies showing diffusion changes in auditory pathways in various diseases accompanied by neurosensory hearing loss are available in the literature $[6,12,14-16]$. In a DTI study performed in patients with unilateral VS, ADC values increased in the contralateral LL, IC, and MGB compared to healthy controls [12]. The authors suggested that high ADC values on the contralateral side may be associated with microstructural changes such as demyelination. They explained that the increase in ADC values on the contralateral side may be due to the natural anatomical course of the auditory pathways [12]. They also found low FA values on both sides in IC. They reported that decreased FA values may be associated with axonal loss, decreased fiber density, and/or demyelination [12]. The changes in ADC and FA values may be attributed to axonal loss and/or demyelination as a result of prolonged injury to the auditory pathways. In 
another study, the authors reported that ADC and FA values in auditory pathways did not change after radiosurgery in patients with VS [6]. They associated it with the presence of the tumor affecting the auditory pathways before radiosurgery. In the current study, we did not detect any changes in ADC and FA values after radiosurgery at LL, IC, and MGB. Our results indicate that auditory pathways may have been influenced by the presence of VS prior to the treatment.

ADC values in contralateral HG increased significantly after radiosurgery. ADC values that increase depending on the delay after radiosurgery may be explained by microstructural alterations such as demyelination. We speculate that the presence of increased ADC values in the contralateral HG may be correlated to the anatomical course of the auditory pathway fibers. Our results support the hypothesis that stimuli from the contralateral side contribute to the processing of auditory stimuli in HG to a greater extent $[6,12]$. In addition, contralateral IC ADC values increase depending on the time after radiosurgery. The presence of increased ADC values at the IC level can be ascribed to the reception of more than one stimulus from the lower auditory pathways on both sides of the brain [5].

The most important parameters in estimating radiation toxicity to the brainstem in radiosurgery treatment are the maximum brainstem dose and BS V10 [14]. In our study, as the radiation dose exposed to the brainstem increased, the contralateral HG FA values decreased slightly. There was no strong correlation between them. A decrease in FA values may be associated with alterations in cytoarchitecture [22]. The weak relationship between the brainstem radiation dose and the slight decrease in contralateral HG FA values may indicate that no significant permanent damage to the radiation-induced auditory pathways had developed.

It is known that an awareness of the mean and maximum cochlear doses is important in the assessment of cochlear toxicity. Studies on GKR have reported that the risk of hearing loss is increased in VS patients who received $>4$ Gy to the cochlea $[3,23,24]$. In our study, the mean cochlear dose was $3.1 \mathrm{~Gy}$, and the mean maximum cochlear dose was $5.8 \mathrm{~Gy}$. In patients with functional hearing, the maximum cochlear dose was kept below $4 \mathrm{~Gy}$ as much as possible. In our study, there was a weak correlation between increased ADC values at ipsilateral MGB and cochlear radiation dose. This weak connection may suggest that increased $\mathrm{ADC}$ values in MGB are unrelated to the cochlear radiation dose.

Recently, diffusion spectrum imaging (DSI), a new diffusion MRI technique, has been developed to noninvasively detect complex white matter pathway architecture and structural changes in fiber pathways in order to overcome the limitations of DTI. Brain reconstruction via DSI shows neural connectivity, such as associative, association, and projection fibers. DSI reconstructs fiber lines with a much higher resolution than DTI and is considered to accurately display transition, winding, and interrupted and small fibers [2527]. Omnidirectional fiber orientation analysis can be avoided using DSI. The general fractional anisotropy (GFA) value is the main quantitative parameter of DSI, representing the directional consistency of water molecule diffusion. DSI is increasingly used nowadays, especially in neuropsychiatric, neurodegenerative, and white matter diseases [25-27].

There are some limitations to our study. Firstly, ROIs were selected for evaluation. When placing ROIs at different locations in the auditory pathways, the problems caused by the partial volume effect can be considered. Secondly, the DTI data of the auditory pathways were not analyzed by tract-based fully automated systems.

\section{Conclusions}

High ADC values in the contralateral HG after radiosurgery may indicate microstructural alterations such as a decrease in the number of axons and an increase in extracellular fluid due to damage to the myelin sheath. Radiation exposure doses to the brainstem and cochlea are the most important factors that can cause microstructural damage to the auditory pathways. We believe that more attention should be paid to prevent the harmful effects of radiation on the auditory pathways when planning radiosurgery. Long-term follow-up and comprehensive DSI studies in the future are needed to analyze in detail the effects of radiosurgery on the auditory pathways in patients with VS and overcome the limitations of DTI.

\section{Additional Information}

\section{Disclosures}

Human subjects: Consent was obtained or waived by all participants in this study. Bezmialem Vakıf University Clinical Research Ethics Committee issued approval 54022451-050.05.04/2020. This study was approved by the Bezmialem Vakıf University Clinical Research Ethics Committee. Animal subjects: All authors have confirmed that this study did not involve animal subjects or tissue. Conflicts of interest: In compliance with the ICMJE uniform disclosure form, all authors declare the following: Payment/services info: All authors have declared that no financial support was received from any organization for the submitted work. Financial relationships: All authors have declared that they have no financial relationships at present or within the previous three years with any organizations that might have an interest in the submitted work. Other relationships: All authors have declared that there are no other 


\section{References}

1. Persson O, Bartek J Jr, Shalom NB, Wangerid T, Jakola AS, Förander P: Stereotactic radiosurgery vs. fractionated radiotherapy for tumor control in vestibular schwannoma patients: a systematic review. Acta Neurochir (Wien). 2017, 159:1013-21. 10.1007/s00701-017-3164-6

2. Buss EJ, Wang TJC, Sisti MB: Stereotactic radiosurgery for management of vestibular schwannoma: a short review (Epub ahead of print). Neurosurg Rev. 2020, 10.1007/s10143-020-01279-2

3. Thomas C, Di Maio S, Ma R, et al.: Hearing preservation following fractionated stereotactic radiotherapy for vestibular schwannomas: prognostic implications of cochlear dose. J Neurosurg. 2007, 107:917-26. 10.3171/JNS-07/11/0917

4. Soltys SG, Milano MT, Xue J, et al.: Stereotactic radiosurgery for vestibular schwannomas: tumor control probability analyses and recommended reporting standards (Epub ahead of print). Int J Radiat Oncol Biol Phys. 2020, 34541-7. 10.1016/j.ijrobp.2020.11.019

5. Chagas Saraiva CW, Cardoso SC, Groppo DP, De Salles AAF, de Ávila LF, Ribeiro da Rosa LA: Gamma Knife radiosurgery for vestibular schwannomas: Evaluation of planning using the sphericity degree of the target volume. PLoS One. 2020, 15:e0225638. 10.1371/journal.pone.0225638

6. Kurtcan S, Hatiboglu MA, Alkan A, et al.: Evaluation of auditory pathways using DTI in patients treated with gamma knife radiosurgery for acoustic neuroma: a preliminary report. Clin Neuroradiol. 2018, 28:377-83. 10.1007/s00062-017-0572-1

7. Boari N, Bailo M, Gagliardi F, et al.: Gamma Knife radiosurgery for vestibular schwannoma: clinical results at long-term follow-up in a series of 379 patients. J Neurosurg. 2014, 121:123-42. 10.3171/2014.8.GKS141506

8. Linskey ME: Hearing preservation in vestibular schwannoma stereotactic radiosurgery: what really matters? J Neurosurg. 2008, 109:129-36. 10.3171/JNS/2008/109/12/S20

9. Paek SH, Chung HT, Jeong SS, et al.: Hearing preservation after gamma knife stereotactic radiosurgery of vestibular schwannoma. Cancer. 2005, 104:580-90. 10.1002/cncr.21190

10. Timmer FC, Hanssens PE, van Haren AE, et al.: Gamma knife radiosurgery for vestibular schwannomas: results of hearing preservation in relation to the cochlear radiation dose. Laryngoscope. 2009, 119:1076-81. 10.1002/lary.20245

11. Han JH, Kim DG, Chung HT, Paek SH, Jung HW: Hearing outcomes after stereotactic radiosurgery for vestibular schwannomas : mechanism of hearing loss and how to preserve hearing. Adv Tech Stand Neurosurg. 2016, 43:3-36. 10.1007/978-3-319-21359-0_1

12. Kurtcan S, Alkan A, Kilicarslan R, et al.: Auditory pathway features determined by DTI in subjects with unilateral acoustic neuroma. Clin Neuroradiol. 2016, 26:439-44. 10.1007/s00062-015-0385-z

13. Bernal B, Altman NR: Auditory functional MR imaging. AJR Am J Roentgenol. 2001, 176:1009-15. 10.2214/ajr.176.4.1761009

14. Lin Y, Wang J, Wu C, Wai Y, Yu J, Ng S: Diffusion tensor imaging of the auditory pathway in sensorineural hearing loss: changes in radial diffusivity and diffusion anisotropy. J Magn Reson Imaging. 2008, 28:598603. 10.1002/jmri.21464

15. Wu CM, Ng SH, Liu TC: Diffusion tensor imaging of the subcortical auditory tract in subjects with long-term unilateral sensorineural hearing loss. Audiol Neurootol. 2009, 14:248-53. 10.1159/000191282

16. Wu CM, Ng SH, Wang JJ, Liu TC: Diffusion tensor imaging of the subcortical auditory tract in subjects with congenital cochlear nerve deficiency. AJNR Am J Neuroradiol. 2009, 30:1773-7. 10.3174/ajnr.A1681

17. Langenhuizen PPJH, Sebregts SHP, Zinger S, Leenstra S, Verheul JB, de With PHN: Prediction of transient tumor enlargement using MRI tumor texture after radiosurgery on vestibular schwannoma. Med Phys. 2020, 47:1692-1701. 10.1002/mp.14042

18. Yomo S, Carron R, Thomassin JM, Roche PH, Régis J: Longitudinal analysis of hearing before and after radiosurgery for vestibular schwannoma. J Neurosurg. 2012, 117:877-85. 10.3171/2012.7.JNS10672

19. Fu VX, Verheul JB, Beute GN, Leenstra S, Kunst HPM, Mulder JJS, Hanssens PEJ: Retreatment of vestibular schwannoma with Gamma Knife radiosurgery: clinical outcome, tumor control, and review of literature. J Neurosurg. 2018, 129:137-45. 10.3171/2017.3.JNS162033

20. Kim JH, Jung HH, Chang JH, Chang JW, Park YG, Chang WS: Predictive factors of unfavorable events after Gamma Knife radiosurgery for vestibular schwannoma. World Neurosurg. 2017, 107:175-84. 10.1016/j.wneu.2017.07.139

21. Meydan S, Aydin S, Otcu H, Kitis S, Alkan A: Assessment of auditory pathways using diffusion tensor imaging in patients with neurofibromatosis type 1. Curr Med Imaging Rev. 2019, 15:890-4. 10.2174/1573405614666180425124743

22. Lin YC, Wang CC, Wai YY, et al.: Significant temporal evolution of diffusion anisotropy for evaluating early response to radiosurgery in patients with vestibular schwannoma: findings from functional diffusion maps. AJNR Am J Neuroradiol. 2010, 31:269-74. 10.3174/ajnr.A1799

23. Tamura M, Carron R, Yomo S, et al.: Hearing preservation after gamma knife radiosurgery for vestibular schwannomas presenting with high-level hearing. Neurosurgery. 2009, 64:289-96. 10.1227/01.NEU.0000338256.87936.7C

24. Hasegawa T, Kato T, Naito T, et al.: Predictors of long-term tumor control after stereotactic radiosurgery for Koos grade 4 vestibular schwannomas. J Neurooncol. 2021, 151:145-56. 10.1007/s11060-020-03622-5

25. Zhang $\mathrm{H}, \mathrm{He}$ WJ, Liang LH, et al.: Diffusion spectrum imaging of corticospinal tracts in idiopathic normal pressure hydrocephalus. Front Neurol. 2021, 12:636518. 10.3389/fneur.2021.636518

26. Zhang Q, Xiao Y, Lin L, Wu J: Diffusion spectrum imaging in white matter microstructure in subjects with type 2 diabetes. PLoS One. 2018, 13:e0203271. 10.1371/journal.pone.0203271

27. Wang YH, Wang ZM, Wei PH, et al.: Lateralizing the affected side of hippocampal sclerosis with quantitative high angular resolution diffusion scalars: a preliminary approach validated by diffusion spectrum imaging. Ann Transl Med. 2021, 9:297. 10.21037/atm-20-5719 